\title{
Plasma collection by an obstacle
}

\author{
H. X. Vu and R. W. Gould \\ Watson Laboratory of Applied Physics, California Institute of Technology, Pasadena, California 91125
}

(Received 22 June 1988; accepted 6 September 1988)

\begin{abstract}
Plasma flow to an obstacle is examined using the two-fluid equations. In this model the obstacle is assumed to be a two-dimensional strip that extends to infinity in the $y$ direction (slab geometry). An obstacle inserted into a magnetized plasma will cast a "shadow" along the magnetic field lines. The natural collection length of such an obstacle is a measure of the length of its shadow. This study shows that in a typical fusion tokamak, where $c_{s} / \Omega_{c i} d \ll 1\left(\Omega_{c i}, c_{s}\right.$ are the ion cyclotron frequency and the ion acoustic speed, respectively, $d$ is the half-width of the strip), the particle collection length of an obstacle can be approximated as $L_{\|}=0.23 c_{s} d^{2} /$ $D_{1}$, if $D_{1} / c_{s} d \ll 1$; or $0.30 d$, if $D_{1} / c_{s} d \gg 1$. For the cases examined in this study, the inclusion of the electron-ion collisional drag parallel to $\mathbf{B}_{0}$ changes the solution only by an insignificant amount.
\end{abstract}

\section{INTRODUCTION}

In fusion tokamaks, limiters are used in order to prevent the vacuum chamber from being bombarded by energetic particles escaping the core plasma, and thereby help reduce the level of impurity in the plasma. It is important to know the plasma density and velocity profiles inside the limitershadow region, and the particle collection length of the limiters. Also, a common density diagnostic consists of a single or an array of Langmuir probes mounted on a probe housing ${ }^{\prime}$ which, in some cases, changes the characteristics of the plasma. It is important to understand how such obstacles perturb the plasma.

Often a qualitative argument based on particle balance ${ }^{2}$ is used to show that a floating obstacle inserted into a magnetized plasma will have a particle collection length along the magnetic field of the order $L_{\|} \sim d_{h}^{2} c_{s} / D_{1}$, where $d_{h}$ and $c_{s}$ are the dimension of the obstacle and the ion acoustic speed, respectively. This paper presents a numerical and analytical study of a simple 2-D model of particle collection by an obstacle. In our model, an obstacle collects ions and electrons at the ion acoustic speed. ${ }^{3-7}$ The external magnetic field is assumed to be uniform and perpendicular to the obstacle. We ignore the effect of an externally applied electric field and mass flow far from the obstacle. Our study shows that for small perpendicular diffusion coefficients, the particle collection length of an obstacle varies directly with the ion acoustic speed, quadratically with the obstacle's dimension, and inversely with the perpendicular diffusion coefficient. For large perpendicular diffusion coefficients, the particle collection length varies directly with the obstacle's dimension and is independent of both the ion acoustic speed and the perpendicular diffusion coefficient. The scaling of the results of our two-dimensional model agrees with the results of the one-dimensional model in Ref. 2 in the limit of small perpendicular diffusion coefficients. In addition, we are able to obtain the numerical constant. We also discuss the case where the perpendicular diffusion coefficient is large, whereas Ref. 2 did not give a corresponding discussion.

\section{MODEL AND ASSUMPTIONS}

Figure 1 illustrates the geometry used in this study. The magnetic field is in the $z$ direction. The obstacle is assumed to be infinitely long in the $y$ direction. Thus $\partial / \partial y=0$. Our domain of interest is $0 \leqslant x \leqslant x_{0}$ and $0 \leqslant z \leqslant z_{0}$. The normal derivative of the density and the normal component of the current $\mathbf{J}$ (in the $x$-z plane) are required to vanish on the boundary of the domain $\left(x=0 ; x=x_{0} ; z=0 ; z=z_{0}\right)$. The normal component of the velocity (in the $x-z$ plane) is also required to vanish on the boundary of the domain except at the obstacle $\left(z=z_{0}, 0 \leqslant x \leqslant d\right)$. There, the normal component of the velocity (the particle collection speed) is taken to be the ion acoustic speed. Below is the list of assumptions employed by our model.

(a) The ions are assumed to be singly charged.

(b) Both the electrons and ions are assumed to be isothermal, and the energy equation is not used.

(c) The pressure is assumed to be isotropic although it may actually be anisotropic due to the presence of an externally applied magnetic field.

(d) Since $m / M \ll 1(m, M$ are the electron's and ion's mass, respectively), the electron inertial term is neglected.

(e) Collisions with neutrals are neglected.

(f) Particles lost to the obstacle are replaced by assuming a spatially uniform source.

(g) The ion Larmor radius is assumed to be small compared to the size of the obstacle.

(h) The Debye length is assumed to be small and we do not treat the sheath regime.

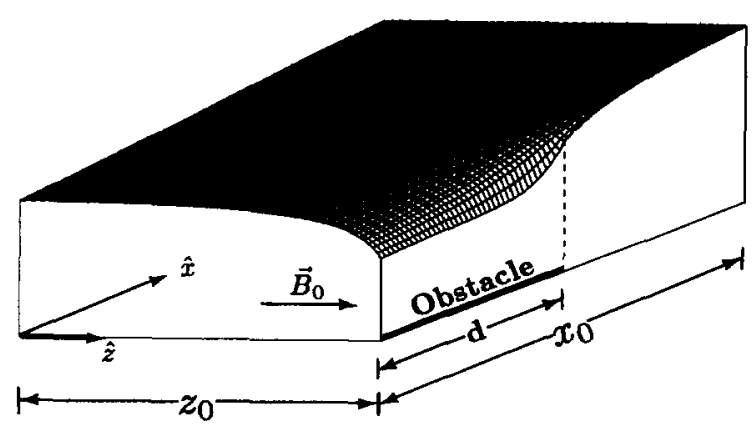

FIG. 1. The density distribution $n(x, z)$ (slab geometry) in the presence of a semi-infinite strip with $c_{s} / \Omega_{c i} d=0.01, D_{\perp} / c_{s} d=\frac{1}{16}, \alpha_{i \mid}=0, z_{0} / d=40$, and $x_{0} / d=2$ is shown. Here, $d$ is the half-width of the obstacle. See that $z_{0} /$ $d$ describes the size of the plasma column compared to the size of the obstacle. 
(i) There is no plasma flow at infinity.

(j) The effect of viscosity is neglected. It will be the subject of a subsequent publication.

We start with the following set of equations:

$$
\begin{aligned}
& M\left(\frac{\partial \mathbf{v}}{\partial t}+(\mathbf{v} \cdot \nabla) \mathbf{v}\right)=\frac{\mathbf{J} \times \mathbf{B}_{0}}{n}-\frac{T_{e}+T_{i}}{n} \nabla n, \\
& 0=-n e\left(-\nabla \phi+\mathbf{u} \times \mathbf{B}_{0}\right)-T_{e} \nabla n \\
& +m v_{\perp} \mathbf{J}_{\perp} / e+m v_{\|} \mathbf{J}_{\|} / e, \\
& \frac{\partial n}{\partial t}+\nabla \cdot(n \mathbf{v})=S, \\
& \boldsymbol{\nabla} \cdot \mathbf{J}=\mathbf{0},
\end{aligned}
$$

where $u$ and $v$ are the electron and ion velocities, respectively, and $\mathbf{J}=n e(v-u)$. Since $m \ll M$, $v$ is essentially the mass velocity. One is tempted to neglect the electron-ion collisional drag parallel to $\mathbf{B}_{0}$ [the last term of Eq. (2)] because then, the electrons would obey a Boltzmann relation on any given field line. This would simplify the equations and their solution significantly. However, a simple estimate shows that the ratio of the electron-ion collisional drag to the electron pressure gradient parallel to $\mathbf{B}_{0}$ is $O\left[\left(v_{\|} / v_{1}\right)\left(1+T_{i} /\right.\right.$ $\left.T_{e}\right)$ ]. Thus it appears necessary to keep the electron-ion collisional drag parallel to $\mathbf{B}_{0}$.

If the electron-ion collisional drag parallel to $\mathbf{B}_{\mathbf{0}}$ were neglected, Eqs. (1)-(3) [with the last term of Eq. (2) removed] would form a closed set of equations, and it would have been unnecessary to include Eq. (4). However, since the electron-ion collisional drag parallel to $\mathbf{B}_{\mathbf{0}}$ is included, we have introduced an additional unknown, namely $\mathbf{J}_{\|}$. Thus Eqs. (1)-(3) no longer form a closed set of equations since we now have more unknowns than we do equations. Therefore, it is necessary to introduce Eq. (4) to close the set of equations.

We define $\psi(x, z)$, the streamfunction for the current $\mathbf{J}$, such that $J_{x}=\partial \psi / \partial z$ and $J_{z}=-\partial \psi / \partial x$. Equation (4) is then automatically satisfied. Since the normal component of $\mathbf{J}$ (in the $x-z$ plane) is required to vanish on the boundary of the domain, $\psi$ is required to be constant on the boundary, which we have chosen to be 0 .

By integrating the $z$ component of Eq. (2) with respect to $z$, and then differentiating it with respect to $x$, one obtains

$$
\begin{aligned}
e \frac{\partial \phi}{\partial x} & -\frac{T_{e}}{n} \frac{\partial n}{\partial x} \\
& =e \frac{d \phi_{0}}{d x}-\frac{T_{e}}{n_{0}} \frac{d n_{0}}{d x}-\frac{m v_{\|}}{e} \frac{\partial}{\partial x} \int_{0}^{z} \frac{J_{z}\left(x, z^{\prime}\right)}{n\left(x, z^{\prime}\right)} d z^{\prime},
\end{aligned}
$$

where $\phi_{0}(x)=\phi(x, 0)$ and $n_{0}(x)=n(x, 0)$. Were it not for the $v_{\|}$term, the density would be related to the potential by the usual Boltzmann factor, i.e., $n(x, z)=n_{0}(x)$ $\times \exp \left[e\left(\phi(x, z)-\phi_{0}(x)\right) / T_{e}\right]$.

Solving for $J_{x}$ from the perpendicular components of Eq. (2), and making use of the above expression and $\mathbf{u}=\mathbf{v}-\mathbf{J} / n e$, one obtains

$$
\begin{aligned}
J_{x}= & \frac{n}{1+\alpha_{\perp}^{2}}\left[e\left(v_{x}+\alpha_{1} v_{y}\right)+\alpha_{1} \alpha_{\|} \frac{\partial}{\partial x}\right. \\
& \left.\times \int_{0}^{z} \frac{J_{z}\left(x, z^{\prime}\right)}{n\left(x, z^{\prime}\right)} d z^{\prime}-\frac{\alpha_{1}}{B_{0}}\left(e \frac{d \phi_{0}}{d x}-\frac{T_{e}}{n_{0}} \frac{d n_{0}}{d x}\right)\right],
\end{aligned}
$$

where $\alpha_{1}=m v_{1} / e B_{0}$ and $\alpha_{\|}=m v_{\|} / e B_{0}$.

Dividing both sides of the equation immediately above by $n$, differentiating the result with respect to $z$, and using the definition for $\psi$, we obtain the following Poisson-like equation for $\psi$ :

$$
\begin{aligned}
(1+ & \left.\alpha_{1}^{2}\right) \frac{\partial}{\partial z}\left(\frac{1}{n} \frac{\partial \psi}{\partial z}\right)+\alpha_{\perp} \alpha_{\|} \frac{\partial}{\partial x}\left(\frac{1}{n} \frac{\partial \psi}{\partial x}\right) \\
& =e \frac{\partial}{\partial z}\left(v_{x}+\alpha_{\perp} v_{y}\right) .
\end{aligned}
$$

Here, $J_{y}$ can be obtained from the perpendicular components of Eq. (2), namely,

$$
J_{y}=\frac{1}{\alpha_{\perp}}\left(J_{x}-n e v_{x}\right)=\frac{1}{\alpha_{1}}\left(\frac{\partial \psi}{\partial z}-n e v_{x}\right) .
$$

In summary, we have kept the electron-ion collisional drag parallel to the external magnetic field $\mathbf{B}_{0}$ in our Ohm's law equation. Since $\boldsymbol{\nabla} \cdot \mathbf{J}=\mathbf{0}$, we have defined a streamfunction $\psi(x, z)$ such that $J_{x}=\partial \psi / \partial z$ and $J_{z}=-\partial \psi / \partial x$. Our model thus consists of Eqs. (1), (5), (6), and (3).

\section{DIMENSIONLESS PARAMETERS AND VARIABLES}

For the sake of simplicity and convenience, we will introduce a set of dimensionless parameters and variables. The dimensionless parameters are $\widetilde{S}=d S / c_{s}$ (normalized ion or electron source), $\rho_{i}=c_{s} / \Omega_{c i} d$, and $\widetilde{D}_{1}=D_{1} / c_{s} d=\alpha_{1} \rho_{i}$ (normalized perpendicular diffusion coefficient), where $T=T_{e}+T_{i}$ and $c_{s}=(T / M)^{1 / 2}$. The dimensionless variables are

$$
\tilde{t}=\frac{c_{s} t}{d}, \quad \tilde{\psi}=\frac{\psi}{d c_{s} e}, \quad \tilde{\mathbf{r}}=\frac{\mathbf{r}}{d}, \quad \tilde{\mathbf{v}}=\frac{\mathbf{v}}{c_{s}}, \quad \tilde{\mathbf{J}}=\frac{\mathbf{J}}{c_{s} e} .
$$

\section{NORMALIZED EQUATIONS}

All of the variables (dependent and independent) will be written without the tilde hereafter. They are understood to be dimensionless. By normalizing Eqs. (1), (5), (6), and (3) one obtains the following set of equations:

$$
\begin{aligned}
& \frac{\partial \mathrm{v}}{\partial t}+(\mathbf{v} \cdot \nabla) \mathbf{v}=\frac{1}{\rho_{i}} \frac{\mathbf{J} \times \hat{z}}{n}-\frac{\nabla n}{n}, \\
& \left(1+\alpha_{1}^{2}\right) \frac{\partial}{\partial z}\left(\frac{1}{n} \frac{\partial \psi}{\partial z}\right)+\alpha_{1} \alpha_{\|} \frac{\partial}{\partial x}\left(\frac{1}{n} \frac{\partial \psi}{\partial x}\right) \\
& \quad=\frac{\partial}{\partial z}\left(v_{x}+\alpha_{1} v_{y}\right), \\
& J_{y}=\frac{1}{\alpha_{1}}\left(J_{x}-n v_{x}\right)=\frac{1}{\alpha_{1}}\left(\frac{\partial \psi}{\partial z}-n v_{x}\right), \\
& \frac{\partial n}{\partial t}+\nabla \cdot(n \mathbf{v})=\widetilde{S},
\end{aligned}
$$

where $\hat{z}$ is the direction of the external magnetic field $\mathbf{B}_{\mathbf{0}}$. 


\section{ANALYTICAL RESULTS}

We have investigated analytically the scaling of an obstacle's particle collection length with $\widetilde{D}_{1}=\alpha_{1} \rho_{i}$. We would like to emphasize that we only consider cases where the ion Larmor radius is small compared to the size of the obstacle. In other words, $\rho_{i}=c_{s} / \Omega_{c i} d \ll 1$. Otherwise, the two-fluid approach is not appropriate. Furthermore, our analytical results are valid only for $\alpha_{\|}=0\left(v_{\|}=0\right)$. With $\alpha_{\|}=0$, it can be shown from Eq. (8) that

$$
\begin{aligned}
J_{x}= & \frac{n}{1+\alpha_{1}^{2}}\left[v_{x}+\alpha_{1} v_{y}-\int_{0}^{z_{11}} n\left(x, z^{\prime}\right)\right. \\
& \left.\times\left[v_{x}\left(x, z^{\prime}\right)+\alpha_{1} v_{y}\left(x, z^{\prime}\right)\right] d z^{\prime}\left(\int_{0}^{z_{0}} n\left(x, z^{\prime}\right) d z^{\prime}\right)^{-1}\right] .
\end{aligned}
$$

Since we are interested in how the parallel collection length scales with $D_{1}$, we restrict ourselves to the case where $z_{0}$ is sufficiently large that the second term of the rhs of the above expression for $J_{x}$ can be neglected compared to the first term. Thus

$$
J_{x}=\left[n /\left(1+\alpha_{1}^{2}\right)\right]\left(v_{x}+\alpha_{\perp} v_{y}\right) .
$$

\section{A. $\tilde{D_{\perp}} \ll 1$}

When $\widetilde{D}_{1}$ and $\rho_{i}$ are much less than unity, it can be shown, by using the perpendicular components of Eq. (7) in steady state $(\partial / \partial t=0)$ and Eqs. (9) and (12), that

$$
\mathbf{v}_{\perp}=\widetilde{D}_{\perp}\left(\nabla_{\perp} n / n\right)-\rho_{i}\left(\nabla_{\perp} n / n\right) \times \hat{z} .
$$

Substituting the $x$ component of the above expression into the continuity equation [Eq. (11)] and renormalizing $z$ by a factor of $\widetilde{D}_{1}$, i.e., letting $z^{*}=\widetilde{D}_{1} z$, one can show that $L_{\|}$, the particle collection length, varies directly with the ion acoustic speed, quadratically with the size of the obstacle, and inversely with the perpendicular diffusion coefficient. Furthermore, the proportionality constant can be estimated in the following manner. In steady state, the rate of particle collection at the obstacle must balance the rate at which particles diffuse across the magnetic field into the collection region that, presumably, extends into the plasma by a distance $L_{\|}$from the obstacle.

\section{B. $\widetilde{D_{1}} \gg 1 \gg p_{i}$}

In this limit, $\alpha_{1}=\widetilde{D}_{1} / \rho_{i} \gg 1$. Equation (13) becomes $J_{x} \simeq 0$. Thus $J_{y} \simeq 0$. The ion momentum equation [Eq. (7)] becomes

$$
\frac{\partial \mathbf{v}}{\partial t}+(\mathbf{v} \cdot \nabla) \mathbf{v}=-\frac{\nabla n}{n} .
$$

In this limit the equations and the boundary conditions do not have any dependence on either $\widetilde{D}_{1}$ or $\rho_{i}$. Therefore, the particle collection length of an obstacle in this limit is independent of both $\widetilde{D}_{1}$ or $\rho_{i}$. Furthermore, it varies directly with the size of the obstacle.

\section{NUMERICAL METHOD}

For the numerical solution of Eqs. (7)-(10) we use the corresponding difference equations on an $81 \times 81$ grid. At the beginning of a time step the streamfunction $\psi$ is computed using successive over relaxation (SOR) using Eq. (8). All time derivatives at the beginning of a time step are evaluated using Eqs. (7) and (10). The velocity and density are advanced half a time step using the Euler method. The streamfunction $\psi$ is computed at the intermediate time step. The time derivatives at the intermediate time step are evaluated using Eqs. (7) and (10). The velocity and density are then advanced an entire time step using time central differences. Equations (7) and (10) are advanced until a steady state is reached. In this scheme, all spatial derivatives are computed using central differences.

\section{NUMERICAL RESULTS}

We have run our 2-D code to obtain the density distribution for various values of $\widetilde{D}_{\perp}$ for both $\alpha_{\|}=0\left(v_{\|}=0\right)$ and $\alpha_{\|}=\alpha_{1}\left(v_{\|}=v_{\perp}\right)$. Figure 1 is a plot of the density distribution $n(x, z)$ (slab geometry) with $\widetilde{D}_{1}=\frac{1}{16}, \rho_{i}=0.01, \alpha_{\|}$ $=0, z_{0} / d=40$, and $x_{0} / d=2$. The density distribution with all parameters being the same except $\alpha_{\|}=\alpha_{1}=6.25$ $\left(v_{\|}=v_{\perp}\right)$ is nearly identical. The density distribution for other values of $\widetilde{D}_{\perp}$ are qualitatively similar. Note that the values of $z_{0} / d$ and $x_{0} / d$ have been chosen large enough that the plasma far from the obstacle is undisturbed, i.e., $n_{0}(x)=$ const.

One can integrate the $z$ component of Eq. (7) with respect to $z$ along $x=0$ (where $v_{x}=0$ ) to show that in steady state $n(x=0, z)=n_{0}(0) \exp \left(-v_{z}^{2} / 2\right)$. Since the normalized particle collection speed at the obstacle is 1 , we have

$$
n\left(x=0, z=z_{0}\right)=n_{0}(0) e^{-1 / 2} \cong 0.6 n_{0}(0) .
$$

We define the particle collection length as the distance (along the obstacle's perpendicular bisector, the line $x=0$ ) from the obstacle where the density is $0.6 n_{0}(0)$ to a point where the density is $0.8 n_{0}(0)$. The normalized particle collection length is defined as the particle collection length nor-

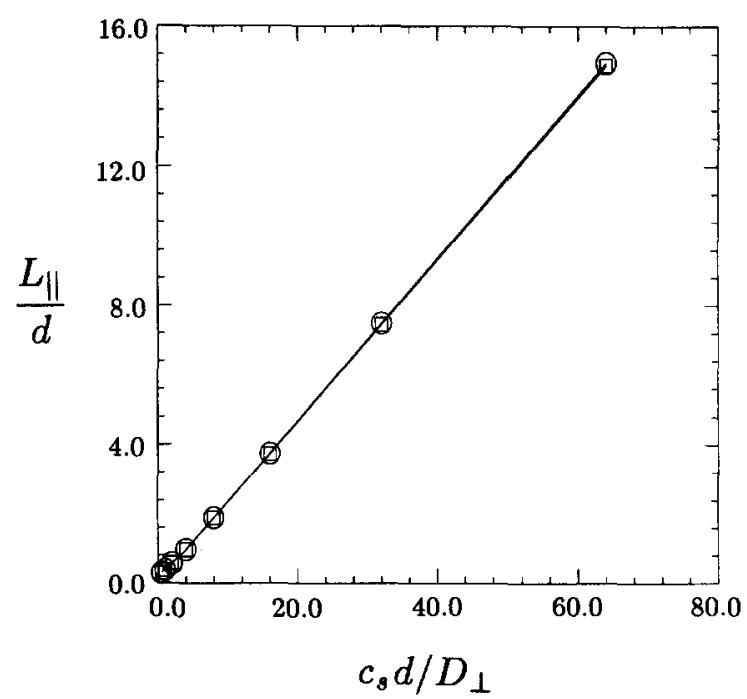

FIG. 2. Plot of the normalized particle collection length $\left(L_{\|} / d\right)$ of a semiinfinite strip versus the inverse of the normalized perpendicular diffusion coefficient $\left(c_{s} d / D_{1}\right)$ for $c_{s} / \Omega_{c i} d=0.01$. The upper and lower curves correspond to $\alpha_{\|}=0$ and $\alpha_{\|}=\alpha_{\downarrow}(O$ and $\square$, respectively). 
malized to $d$, the half-width of the semi-infinite strip. Figure 2 is a plot of $L_{\|} / d$ (the normalized particle collection length of the strip) versus $c_{s} d / D_{\perp}$ (the inverse of the normalized perpendicular diffusion coefficient), for $\rho_{i}=0.01$. The upper and lower curves correspond to $\alpha_{\|}=0$ and $\alpha_{\|}=\alpha_{1}$ for the semi-infinite strip ( $O$ and $\square$, respectively). The analytical analysis predicts that for $\widetilde{D}_{1}, \rho_{i} \ll 1$, the plot of $L_{\|} / d$ vs $c_{s} d / D_{1}$ should be a straight line. For $\widetilde{D}_{1} \gg 1 \gg \rho_{i}$, the prediction is that $L_{\|} / d$ should approach a constant (independent of $\left.\widetilde{D}_{1}\right)$. The upper curve $\left(\alpha_{\|}=0\right)$ shows that this is indeed the case, and that for $\widetilde{D}_{1} \leqslant 1$, the plot of $L_{\|} / d$ vs $c_{s} d / D_{1}$ approaches a straight line with a slope of about 0.23 . A comparison of the upper $\left(\alpha_{\|}=0\right)$ and lower $\left(\alpha_{\|}=\alpha_{1}\right)$ curves shows that the inclusion of the electron-ion collisional drag parallel to $\mathbf{B}_{0}$ made an insignificant contribution to the solution.

\section{SUMMARY AND CONCLUSIONS}

In this paper we have performed both a numerical and analytical study of a simple 2-D model describing plasma collection by an obstacle. In our model we ignore the effect of an external electric field and mass flow far from the obstacle. An obstacle is assumed to collect ions and electrons at the ion acoustic speed. The external magnetic field is assumed to be uniform and perpendicular to the obstacles. Our study shows that in a typical fusion tokamak, where $c_{s} / \Omega_{c i} d \leqslant 1$, the particle collection length of an obstacle can be approximated as follows:

$$
L_{\|}= \begin{cases}0.23 c_{s} d^{2} / D_{\perp}, & \text { if } D_{\perp} / c_{s} d<1, \\ 0.30 d, & \text { if } D_{\perp} / c_{s} d>1\end{cases}
$$

Our study also shows that, although the electron-ion collisional drag parallel to the external magnetic field $\mathbf{B}_{0}$ can be (analytically) shown to be of the order of the electron pressure gradient parallel to $\mathbf{B}_{\mathbf{0}}$, its inclusion in the cases we have examined only changes the solution by an insignificant amount.

\section{ACKNOWLEDGMENTS}

This work was supported by the U.S. Department of Energy, Office of Fusion Energy Grant No. DE-FG0385ER53173 and the Magnetic Fusion Science Fellowship Program.

'See, for example, S. J. Zweben and R. W. Gould, Nucl. Fusion 25, 171 (1985).

${ }^{2}$ P. C. Stangeby, J. Phys. D 18, 1547 (1985).

${ }^{3} \mathrm{D}$. Bohm, in The Characteristics of Electrical Discharges in Magnetic Fields, edited by A. Guthrie and R. K. Wakerling (McGraw-Hill, New York, 1949), Chap. 3.

${ }^{4}$ E. R. Harrison and W. B. Thompson, Proc. Phys. Soc. 74, 145 (1959).

${ }^{5}$ S. A. Self, Phys. Fluids 6, 1762 (1963).

${ }^{6}$ P. C. Stangeby and J. E. Allen, J. Phys. A 3, 304 (1970).

${ }^{7}$ J. E. Allen, J. Phys. D 9, 2331 (1976). 